1 Universidade Federal do Espírito Santo (Ufes) Vitória (ES), Brasil. cmlf.odontologia@gmail.com

2 Fundação Oswaldo Cruz (Fiocruz), Escola Naciona de Saúde Pública Sergio Arouca (Ensp), Programa de Pós-Graduação em Saúde Pública - Rio de Janeiro (RJ), Brasil.

\section{Tempo de espera e absenteísmo na atenção especializada: um desafio para os sistemas universais de saúde}

\author{
Waiting time and absenteeism in the secondary care: a challenge for \\ universal health systems
}

Cynthia Moura Louzada Farias', Ligia Giovanella², Adauto Emmerich Oliveira', Edson Theodoro dos Santos Neto'

DOI: $10.1590 / 0103-11042019 S 516$

RESUMO O objetivo do estudo foi analisar o absenteísmo em relação ao tempo de espera por consultas e exames especializados nos 20 municípios que compõem a Região de Saúde Metropolitana do estado do Espírito Santo (RSM-ES), Brasil. Estudo descritivo retrospectivo realizado a partir da análise do Banco de Dados da Secretaria de Estado da Saúde do Espírito Santo (Sesa). Foram considerados 1.002.719 encaminhamentos dos usuários residentes na RSM-ES para consultas/exames especializados fora do município no período de janeiro de 2014 a dezembro de 2016, que correspondem a todos os agendamentos. O tempo médio de espera pela consulta foi de 419 dias (desvio padrão $=29,3$, mediana $=17,0$ ) em 2014, de 687 dias (desvio padrão $=70,5$, mediana $=16,0$ ) em 2015, de 1.077 dias (desvio padrão = 140,3, mediana = 20,0) em 2016, aumento progressivo da espera com o passar dos anos. As análises de correlação do estudo apontaram que o tempo de espera e o porte municipal são fatores correlacionados às taxas de absenteísmo em consultas e exames especializados ( $\mathrm{p}$-valor $<5 \%$ ). O impacto do absenteísmo nos serviços ambulatoriais, influenciado pelo tempo de espera, constitui-se em um grande desafio para a estruturação de um sistema público de saúde no Brasil. Conhecer como certos fatores impactam o comportamento de não comparecimento a compromissos agendados em municípios pode subsidiar mudanças nas políticas de agendamento de consultas/exames especializados.

PALAVRAS-CHAVE Listas de espera. Acesso aos serviços de saúde. Serviços de saúde. Atenção secundária à saúde.

ABSTRACT The study aimed to analyze the waiting time for specialized consultations and examinations in the twenty municipalities that compose the Metropolitan Health Region of Espírito Santo State (RSM-ES), Brazil. It is a retrospective and descriptive study was carried out based on the analysis of the Database of the State Health Department of Espírito Santo (Sesa). A total of 1,002,719 referrals from users residing in the RSM-ES were considered for specialized consultations/exams outside the municipality from January 2014 to December 2016, which corresponds to all schedulings. The average waiting time for the consultation was 419 days (standard deviation $=29.34$, median $=17.00$ ) in 2014, 687 days (standard deviation $=70.51$, median $=16.00)$ in 2015,1077 days (standard deviation $=140.26$, median $=20.00$ ) in 2016, progressive increase in waiting over the years. The correlation analyses of the study indicate that waiting time and municipal size are factors that can influence absenteeism in specialized consultations and examinations. The impact of absenteeism on outpatient services, influenced by waiting time, constitutes a major challenge for the structuring of a public health system in Brazil. Knowing how certain factors impact the behavior of non-attendance to appointments scheduled in municipalities can substantiate changes in scheduling policies for specialized consultations/examinations.

KEYWORDS Waiting lists. Health services accessibility. Health services. Secondary care. 


\section{Introdução}

O crescimento da demanda por procedimentos médicos especializados é um problema corrente nos sistemas públicos de saúde ${ }^{\mathbf{1 , 2}}$. Como resultado, é comum que existam listas de espera extensas, que resultam em um tempo de espera de meses (ou até mesmo anos) para a avaliação de um usuário por especialistas ${ }^{3}$. Isso gera atrasos na realização de diagnósticos, diminuição na efetividade de muitos tratamentos, faltas aos procedimentos agendados e ansiedade para o usuário aguardando ${ }^{4}$.

A magnitude dos tempos de espera para procedimentos eletivos é um problema em cerca da metade dos países da Organização para a Cooperação e o Desenvolvimento Econômico $(\mathrm{OCDE})^{\mathbf{5}}$, mas também ocorre em sistemas com formas de organização distintas e predomínio de prestadores privados, como é o caso da Austrália, do Canadá e da Nova Zelândia ${ }^{6}$. No Brasil, os elevados tempos de espera para marcação de consultas, exames especializados e cirurgias constituem a maior causa de insatisfação referida pelos usuários do Sistema Único de Saúde (SUS)7,8.

O tempo de espera pelo atendimento pode ter impacto na evolução dos casos, influenciando o prognóstico e a qualidade de vida de pacientes com doenças graves, sintomáticas ou estigmatizantes. Além disso, ele é um indicador da qualidade dos serviços, por estar relacionado com a capacidade de resposta do sistema às necessidades de atenção à saúde da população. Um tempo de espera longo diminui a produtividade e a eficiência, aumenta os custos com saúde e limita a capacidade efetiva da clínica de saúde ${ }^{9-12}$. Entre várias situações, imprecisões no planejamento na gestão da relação oferta/demanda de serviços podem interferir no tempo de espera e absenteísmo em consultas e exames especializados ${ }^{13}$.

De modo geral, os usuários podem encontrar três tipos de espera durante a sua interação com a atenção especializada de saúde. A primeira espera é o intervalo entre o momento em que o profissional faz um encaminhamento e a visita real do usuário ao especialista. O especialista pode solicitar exames adicionais (como uma ressonância magnética) como um prelúdio para o tratamento, e o usuário pode então encontrar uma terceira espera (pela ressonância magnética e/ou tratamento) ${ }^{8}$. O segundo intervalo corresponde ao tempo entre o encaminhamento e a espera pelo agendamento do procedimento especializado. Já o terceiro tempo compreende o período entre o agendamento e a execução da consulta ou exame especializado.

No Brasil, o longo tempo de espera para consultas especializadas está entre as principais barreiras ao acesso a cuidados integrais à saúde no SUS ${ }^{8}$. O aumento da demanda, sobretudo decorrente da ampliação da cobertura da atenção básica, do aumento da expectativa de vida e da prevalência de doenças crônicas, somado à insuficiência de recursos e de serviços, tem dificultado o acesso à atenção especializada ${ }^{\mathbf{1 4}, 15}$.

Nesse contexto, se esse problema ocorre em grandes municípios ${ }^{8,15}$, nos pequenos, distantes de grandes centros urbanos e, por vezes, localizados em verdadeiros vazios assistenciais, a situação pode ser mais grave ${ }^{14,16}$. Ainda, se esses municípios estiverem localizados em regiões pouco desenvolvidas economicamente, $\mathrm{o}$ acesso a consultas com médicos especialistas chega a ser considerado um verdadeiro desafio ${ }^{17}$.

Assim, reduzir os tempos de espera pelos cuidados de saúde é um dos principais desafios dos gestores e formuladores de políticas públicas no Brasil. Mesmo que a maioria das necessidades em saúde possa ser satisfatoriamente resolvida pela equipe da atenção primária ${ }^{8}$, são necessários aparatos especializados para garantir a continuidade do cuidado para efetivar o princípio da integralidade no nível de atenção secundaria ${ }^{18}$. Além disso, torna-se um desafio sobretudo para um sistema de políticas universais, tanto em sua capacidade de responder às demandas e necessidades atuais quanto na sua necessária sustentabilidade no futuro.

Diante da relevância e da escassez de estudos que tratam do nível de atenção especializada no SUS, este estudo se propõe analisar o absenteísmo e a relação com os tempos de 
espera por consultas e exames especializados na Região de Saúde Metropolitana do estado do Espírito Santo (RSM-ES), Brasil.

\section{Métodos}

Trata-se de um estudo descritivo e retrospectivo realizado a partir da análise do Banco de Dados da Secretaria de Estado da Saúde do Espírito Santo (Sesa). Como fonte de dados deste estudo, foram considerados 1.002.719 encaminhamentos dos usuários residentes na RSM-ES para consultas/exames especializados fora do município no período de janeiro de 2014 a dezembro de 2016, que correspondem a todos os agendamentos.

A RSM-ES é composta por 20 municípios e possui população total estimada de 2.180 .633 habitantes, sendo que $55 \%$ dessa população se concentra em área urbana. Entre eles, sete municípios têm mais de $50 \%$ de sua população na área rural. A RSM-ES representa $55 \%$ da população total do Espírito Santo ${ }^{19}$.

A rede básica de serviços de saúde da região do estudo está estruturada na Estratégia Saúde Família; e no que se refere ao acesso aos serviços especializados, os municípios da região estão inseridos em uma configuração de rede de serviços de saúde regionalizada determinada pelo Plano Diretor de Regionalização (PDR) do Espírito Santo em 2011. O modelo de atenção preconizado para o SUS deve garantir a integralidade a partir da consolidação do papel destinado a atenção básica como ordenador da rede e coordenador do cuidado. Isso significa que o acesso aos demais níveis de atenção deve ser orientado pela regulação realizada nas unidades básicas de saúde. Entretanto, atualmente, para os usuários que necessitam de consultas médicas de especialidades não ofertadas no município, o acesso se dá pelo Sistema de Regulação de Consultas e Exames (SisReg). Para tal, o fluxo inicia-se com o preenchimento de encaminhamento específico pelo médico solicitante na unidade de saúde no município de residência do usuário.
Em seguida, o usuário recebe o encaminhamento e o entrega na Central Municipal de Marcação de Consultas, onde um profissional se responsabiliza pelo agendamento da consulta por meio de contatos com a Central de Regulação Estadual. Entre as variáveis disponíveis do banco do SisReg, para fins deste estudo, foram utilizadas somente as variáveis: município residência do usuário, porte populacional municipal, data de solicitação, data de autorização, data de execução, número de consulta e exames confirmados e não confirmadas (absenteísmo).

A análise do tempo de espera em consultas/ exames especializados foi realizada a partir do porte populacional dos municípios que compõem o estado do ES por meio das faixas populacionais de: até 10.000 habitantes; de 10.001 a 20.000; de 20.001 a 50.000; de 50.001 a 200.000; 200.001 habitantes ou mais, as quais tiveram como referência a divisão de porte populacional utilizada pelo Instituto Brasileiro de Geografia e Estatística (IBGE), modificadas de acordo com a realidade do $\mathrm{ES}^{\mathbf{2 0}}$. Sendo que o município mais populoso do estado é o de Serra com 507.598 habitantes.

A análise correspondeu ao tempo de espera para execução de consultas/exames especializados agendados por usuários residentes na RSM-ES, agendamentos que compreendem consultas/exames especializados não ofertadas em seu município de residência.

O tempo de espera ( $\mathrm{T}$ ) para esse fluxo que foi dividido em três categorias: a diferença entre a data de solicitação da consulta e exame especializado e a data da autorização da consulta (T1); a data de autorização da consulta e exame especializado e a data da execução da consulta e exame especializado (T2); e a data de solicitação da consulta e exame especializado e a data da execução da consulta e exame especializado (T3). A solicitação corresponde à inclusão do usuário na fila de potencial agendamento. A autorização significa que o compromisso de atendimento foi firmado e que a data será disponibilizada ao usuário. A execução corresponde ao atendimento de fato realizado, conforme explicitado na figura 1. 
Figura 1. Categorias do tempo de espera em consultas e exames especializados

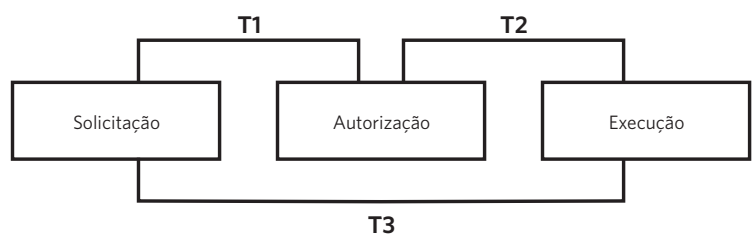

Fonte: Elaboração própria.

A análise estatística foi realizada por meio do programa Microsoft Office Excel ${ }^{\circledR} 2010$ e Statistical Package for the Social Sciences, versão 20.0. Depois de analisar o banco de dados, prosseguiu-se a análise descritiva. Os resultados são expressos como mediana, média, desvio padrão. A análise de correlação de Sperman considerou o tempo de espera, a taxa de absenteísmo por município e o porte municipal, com um nível de significância de $5 \%$. O estudo foi autorizado pela Secretaria Estadual de Saúde e aprovado pelo Comitê de Ética em Pesquisa do Centro de Ciências da Saúde da Universidade Federal do Espírito Santo (Ufes) sob o parecer $n^{\circ}$ CAAE 52838416.0.0000.5060, atendendo à Resolução $n^{0} 466 / 2012$.

\section{Resultados}

No período de janeiro de 2014 a dezembro de 2016, foram registrados os dados acerca do tempo de espera e das faltas dos usuários a consultas/exames especializados agendados. Para as consultas especializadas no ano de 2014, em um total de agendadas de 200.831, o absenteísmo foi de 74.126 (36,9\%); em 2015, em um total de 225.818 agendamentos a consultas, 87.492 (38,7\%) não compareceram; e em 2016, em um total de 239.533 consultas agendadas, registrou-se o absenteísmo de 95.407 (39,8\%), sendo que nos três anos o absenteísmo registrado foi de $38,6 \%$ (257.025 consultas). Para os exames especializados, foi constatado que a taxa de absenteísmo vem decrescendo anualmente, sendo 34,3\% em 2014, 32,7\% em 2015 e $29,7 \%$ em 2016. A taxa total de absenteísmo para os exames especializados foi de $32,1 \%$ (108.103 exames). Os usuários faltosos foram separados por ano e seus respectivos valores percentuais em consultas/exames.

O estudo permitiu verificar que $50 \%$ dos usuários esperaram até 8 dias para realizar consultas/exames especializados, entretanto, outros $50 \%$ podem aguardar até 1.077 dias. É importante ressaltar que as médias não estão representando o universo analisado, pois a variabilidade representada pelo desvio-padrão apresenta-se muito grande. Os menores valores nos tempos de espera variaram de 0 a 1 dia, ou seja, alguns usuários solicitam e conseguem realizar sua consulta no mesmo dia do agendamento. As medianas dos tempos de espera são muito semelhantes em relação aos períodos analisados.

Quanto à localização do domicílio, o tempo máximo de espera registrado para os procedimentos especializados foi de 1.077 dias no município de Marechal Floriano, seguido de 1.056 dias no município de Cariacica. O tempo médio de espera por procedimentos, independentemente da especialidade, foi de 419 dias com (desvio padrão $=29,34$, mediana $=17,00$ ) em 2014, de 687 dias (desvio padrão $=70,51$, mediana $=16,00$ ) em 2015, de 1.077 dias (desvio padrão $=140,26$, mediana $=20,00$ ) em 2016, com coeficiente de variação crescente ou aumento progressivo da espera com o passar dos anos para as consultas especializadas. 
Em relação às esperas em T1, o maior valor foi registrado em 2014 e 2016, respectivamente, no município de Marechal Floriano (409 e
1.054 dias). Em 2015, o município de Cariacica registrou tempo de espera em $\mathrm{T} 1$ de 678 dias. Esses valores são apresentados na tabela 1 .

Tabela 1. Distribuição do tempo entre solicitação e autorização (em dias) das consultas e exames especializados. RSM-ES, Brasil. 2014-2016

\begin{tabular}{|c|c|c|c|c|c|c|c|c|c|c|c|c|c|c|}
\hline \multirow{2}{*}{\multicolumn{2}{|c|}{ Porte Município }} & \multirow[b]{2}{*}{ População } & \multicolumn{4}{|c|}{$2014(n=305.083)$} & \multicolumn{4}{|c|}{$2015(n=340.177)$} & \multicolumn{4}{|c|}{$2016(n=357.459)$} \\
\hline & & & Mediana & Maior & Média & Desvio- & Mediana & Maior & Média & Desvio- & Mediana & Maior & Média & Desvio- \\
\hline$A$ & Brejetuba & 12.381 & 1 & 234 & 13,68 & 27,7 & 0 & 518 & 25,72 & 62,39 & 3,5 & 730 & 36,5 & 90,01 \\
\hline$A$ & Conceição do Castelo & 12.638 & 0 & 392 & 10,46 & 26,12 & 0 & 608 & 18,23 & 57,94 & 0 & 647 & 21,03 & 75,8 \\
\hline$A$ & Itaguaçu & 14.109 & 2 & 239 & 19,04 & 33,89 & 0 & 523 & 24,99 & 66,39 & 0 & 768 & 35,7 & 94,32 \\
\hline$A$ & Itarana & 10.619 & 5 & 268 & 19,97 & 33 & 0 & 507 & 28,42 & 70,8 & 1 & 790 & 47,9 & 115,21 \\
\hline$A$ & Laranja da Terra & 10.961 & 4 & 225 & 23,98 & 43,27 & 10 & 580 & 55,49 & 89,94 & 13 & 783 & 95,01 & 162,57 \\
\hline$A$ & Marechal Floriano & 16.464 & 0 & 409 & 11,1 & 24,36 & 0 & 572 & 29,78 & 75,29 & 0 & 1054 & 29,07 & 101,3 \\
\hline$A$ & Santa Leopoldina & 12.300 & 0 & 285 & 6,92 & 22,53 & 0 & 517 & 13,28 & 57,36 & 0 & 886 & 19,94 & 74,39 \\
\hline B & Fundão & 21.061 & 0 & 268 & 5,45 & 21,65 & 0 & 402 & 8,01 & 35,85 & 0 & 860 & 13,34 & 51,47 \\
\hline B & Ibatiba & 25.732 & 0 & 282 & 11,45 & 28,69 & 0 & 498 & 22,76 & 64,39 & 0 & 873 & 23,26 & 78,8 \\
\hline B & Santa Teresa & 23.392 & 0 & 358 & 10,99 & 26,53 & 0 & 593 & 25,08 & 65,04 & 0 & 854 & 27,45 & 82,48 \\
\hline B & Venda Nova do Imigrante & 24.800 & 8 & 304 & 25,78 & 37,55 & 22 & 535 & 87,49 & 122,19 & 14 & 936 & 81,5 & 145,58 \\
\hline B & Afonso Cláudio & 30.720 & 1 & 271 & 15,91 & 33,08 & 0 & 602 & 26,79 & 67,58 & 3 & 931 & 77,02 & 158,84 \\
\hline B & Domingos Martins & 33.711 & 0 & 341 & 15,94 & 31,31 & 0 & 664 & 39,06 & 87,6 & 0 & 899 & 30,63 & 79,8 \\
\hline B & Santa Maria de Jetibá & 39.849 & 1 & 409 & 17,59 & 35,5 & 0 & 553 & 24,02 & 65,49 & 1 & 1006 & 47,69 & 107,91 \\
\hline C & Viana & 76.954 & 0 & 238 & 8,23 & 24,03 & 0 & 600 & 14,86 & 49,74 & 1 & 976 & 47,05 & 100,14 \\
\hline C & Guarapari & 122.982 & 3 & 340 & 23,08 & 45,15 & 0 & 591 & 30,91 & 70,99 & 20 & 1050 & 169,87 & 236,16 \\
\hline $\mathrm{D}$ & Cariacica & 378.603 & 0 & 401 & 11,11 & 28,34 & 4 & 678 & 50,25 & 83,53 & 5 & 1047 & 103,19 & 177,66 \\
\hline D & Vitória & 358.267 & 0 & 406 & 10,58 & 30,62 & 0 & 637 & 28,92 & 66,15 & 0 & 929 & 52,61 & 125,7 \\
\hline $\mathrm{D}$ & Vila Velha & 486.208 & 0 & 288 & 8,84 & 24,7 & 0 & 651 & 17,98 & 61,84 & 0 & 882 & 41,59 & 121,36 \\
\hline $\mathrm{D}$ & Serra & 507.598 & 0 & 317 & 5,36 & 19,8 & 0 & 669 & 14,04 & 58,51 & 0 & 857 & 38,89 & 106,96 \\
\hline \multicolumn{2}{|c|}{ RSM-ES } & 2.219 .349 & 0 & 409 & 10,31 & 28,28 & 0 & 678 & 27,44 & 69,3 & 0 & 1054 & 59,59 & 139,1 \\
\hline
\end{tabular}

Fonte: Elaboração própria.

A: Até 20.000 habitantes; B: 20.001 a 50.000 habitantes; C: 50.001 a 200.000 habitantes; D: Acima de 200.001 habitantes.

As maiores esperas em $\mathrm{T} 2$ foram de 90 dias em 2014 nos municípios de Cariacica, Vitória, Serra e Marechal Floriano; de 87 dias em 2015 e de 77 dias em 2016 no município de Cariacica. O estudo permitiu verificar que, em T2, 50\% dos usuários esperam até 15 dias entre a autorização e a execução de sua consulta ou exame especializado, mas metade deles esperam até 90 dias, demonstrados na tabela 2 . 
Tabela 2. Distribuição do tempo entre autorização e execução (em dias) das consultas e exames especializados. RSM-ES, Brasil. 2014-2016

\begin{tabular}{|c|c|c|c|c|c|c|c|c|c|c|c|c|c|c|}
\hline & & & & $014(n=3$ & 305.083) & & & $015(n=3$ & 340.177) & & & $016(n=$ & 357.459) & \\
\hline Por & Município & População & Mediana & Maior & Média & Desvio- & Mediana & Maior & Média & Desvio- & Mediana & Maior & Média & Desvio- \\
\hline$A$ & Brejetuba & 12.381 & 15 & 42 & 14,66 & 6,01 & 14 & 48 & 14,39 & 5,38 & 15 & 36 & 15,75 & 5,98 \\
\hline A & Conceição do Castelo & 12.638 & 14 & 43 & 13,43 & 6,4 & 14 & 56 & 13,12 & 5,6 & 14 & 39 & 14,32 & 5,75 \\
\hline A & Itaguaçu & 14.109 & 16 & 45 & 16,16 & 6,14 & 14 & 52 & 15,04 & 4,87 & 14 & 37 & 15,7 & 5,65 \\
\hline A & Itarana & 10.619 & 17 & 44 & 16,14 & 6,12 & 14 & 35 & 14,97 & 5,2 & 17 & 56 & 16,42 & 5,63 \\
\hline$A$ & Laranja da Terra & 10.961 & 16 & 43 & 15,82 & 5,74 & 16 & 30 & 16,07 & 5,73 & 19 & 38 & 17,04 & 5,66 \\
\hline A & Marechal Floriano & 16.464 & 15 & 90 & 15,33 & 6,74 & 14 & 41 & 13,76 & 5,03 & 14 & 70 & 15,04 & 5,35 \\
\hline$A$ & Santa Leopoldina & 12.300 & 15 & 65 & 15,83 & 6,86 & 14 & 52 & 13,86 & 5,15 & 14 & 43 & 15,03 & 5,47 \\
\hline B & Fundão & 21.061 & 15 & 44 & 14,56 & 5,83 & 14 & 52 & 14,25 & 4,3 & 14 & 68 & 15,65 & 5,02 \\
\hline B & Ibatiba & 25.732 & 15 & 85 & 14,83 & 6,84 & 14 & 24 & 14,93 & 5,34 & 16 & 42 & 15,8 & 5,56 \\
\hline B & Santa Teresa & 23.392 & 15 & 56 & 15,4 & 6,87 & 14 & 42 & 14,11 & 5,66 & 17 & 38 & 16,46 & 5,73 \\
\hline B & Venda Nova do Imigrante & 24.800 & 16 & 84 & 15,59 & 6,87 & 16 & 33 & 15,66 & 5,88 & 19 & 43 & 17,51 & 5,79 \\
\hline B & Afonso Cláudio & 30.720 & 16 & 53 & 16,39 & 6,62 & 14 & 52 & 15,03 & 5,37 & 18 & 68 & 16,43 & 5,51 \\
\hline B & Domingos Martins & 33.711 & 16 & 77 & 16,55 & 6,94 & 17 & 48 & 15,96 & 5,16 & 17 & 38 & 16,7 & 5,26 \\
\hline B & Santa Maria de Jetibá & 39.849 & 15 & 66 & 14,87 & 6,9 & 14 & 52 & 14,21 & 5,25 & 15 & 44 & 16,22 & 5,7 \\
\hline C & Viana & 76.954 & 15 & 77 & 14,32 & 5,42 & 14 & 56 & 14,16 & 4,57 & 17 & 68 & 16,43 & 5,52 \\
\hline C & Guarapari & 122.982 & 17 & 48 & 16,32 & 5,72 & 15 & 69 & 15,21 & 5,43 & 20 & 69 & 17,5 & 5,54 \\
\hline D & Cariacica & 378.603 & 16 & 90 & 16,09 & 5,92 & 20 & 87 & 16,78 & 5,26 & 18 & 77 & 16,43 & 5,93 \\
\hline D & Vitória & 358.267 & 15 & 90 & 15,63 & 6,66 & 15 & 68 & 15,59 & 5,77 & 14 & 76 & 15,5 & 6,01 \\
\hline $\mathrm{D}$ & Vila Velha & 486.208 & 15 & 86 & 14,98 & 5,54 & 14 & 35 & 15,32 & 4,94 & 14 & 72 & 16,33 & 6,15 \\
\hline D & Serra & 507.598 & 14 & 90 & 14,54 & 6,38 & 14 & 75 & 14,06 & 4,93 & 14 & 75 & 14,89 & 5,52 \\
\hline & & 2.219 .349 & 15 & 90 & 15,38 & 6,25 & 14 & 87 & 15,28 & 69,3 & 14 & 77 & 15,9 & 5,87 \\
\hline
\end{tabular}

Fonte: Elaboração própria.

A: Até 20.000 habitantes; B: 20.001 a 50.000 habitantes; C: 50.001 a 200.000 habitantes; D: Acima de 200.001 habitantes.

As esperas mais longas foram em T3, respectivamente: de 419 dias em 2014 nos municípios de Marechal Floriano e Santa Maria de Jetibá, de 687 dias em 2015 em Cariacica e de 1.077 dias em 2016 no município de Marechal
Floriano. Os maiores tempos de espera e diferenças maiores foram observadas em $\mathrm{Tl}$ e T3. O menor valor para todos os municípios foi zero em todos os anos. Esses dados são demostrados na tabela 3. 
Tabela 3. Tempo entre solicitação e execução (em dias) das consultas e exames especializados. RSM-ES, Brasil. 2014-2016

\begin{tabular}{|c|c|c|c|c|c|c|c|c|c|c|c|c|c|c|c|}
\hline \multirow[b]{2}{*}{ Porte } & \multirow[b]{2}{*}{ Município } & \multirow[b]{2}{*}{ População } & \multicolumn{4}{|c|}{$2014(n=305.083)$} & \multicolumn{4}{|c|}{$2015(n=340.177)$} & \multicolumn{5}{|c|}{$2016(n=357.459)$} \\
\hline & & & $\begin{array}{r}\text { Taxa de } \\
\text { Absente- } \\
\text { ísmo }\end{array}$ & $\begin{array}{l}\text { Maior } \\
\text { Valor }\end{array}$ & Média & $\begin{array}{l}\text { Desvio- } \\
\text { padrão }\end{array}$ & $\begin{array}{r}\text { Taxa de } \\
\text { Absente- } \\
\text { ísmo }\end{array}$ & $\begin{array}{l}\text { Maior } \\
\text { Valor }\end{array}$ & Média & $\begin{array}{l}\text { Desvio- } \\
\text { padrão }\end{array}$ & $\begin{array}{r}\text { Taxa de } \\
\text { Absente- } \\
\text { ísmo }\end{array}$ & Mediana & $\begin{array}{l}\text { Maior } \\
\text { Valor }\end{array}$ & Média & $\begin{array}{l}\text { Desvio- } \\
\text { padrão }\end{array}$ \\
\hline$A$ & Brejetuba & 12.381 & 26,6 & 248 & 28,34 & 28,57 & 33 & 542 & 40,11 & 63,59 & 36,4 & 21 & 756 & 52,24 & 91,26 \\
\hline A & $\begin{array}{l}\text { Conceição do } \\
\text { Castelo }\end{array}$ & 12.638 & 19.6 & 412 & 23,89 & 27,55 & 26,4 & 629 & 31,36 & 59,22 & 32,5 & 14 & 668 & 35,35 & 77,34 \\
\hline A & Itaguaçu & 14.109 & 23,5 & 252 & 35,2 & 34,3 & 27,4 & 540 & 40,03 & 67,51 & 31,9 & 20 & 789 & 51,4 & 94,95 \\
\hline A & Itarana & 10.619 & 24,7 & 273 & 36,1 & 33,67 & 29,9 & 529 & 43,39 & 71,74 & 33 & 20 & 804 & 64,32 & 115,8 \\
\hline A & Laranja da Terra & 10.961 & 38,7 & 240 & 39,8 & 43,77 & 40,2 & 604 & 71,56 & 91,15 & 35,7 & 32 & 794 & 112,04 & 163,26 \\
\hline A & $\begin{array}{l}\text { Marechal Flo- } \\
\text { riano }\end{array}$ & 16.464 & 25,1 & 419 & 26,43 & 25,89 & 32,4 & 593 & 43,54 & 76,63 & 33,5 & 14 & 1077 & 44,1 & 102,26 \\
\hline A & Santa Leopoldina & 12.300 & 23,1 & 301 & 22,74 & 23,58 & 26,8 & 539 & 27,13 & 58,3 & 29,2 & 14 & 907 & 34,97 & 75,29 \\
\hline B & Fundão & 21.061 & 31,9 & 280 & 20,01 & 22,47 & 33,3 & 425 & 22,26 & 36,61 & 32,7 & 14 & 881 & 28,98 & 52,4 \\
\hline B & Ibatiba & 25.732 & 26,2 & 302 & 26,28 & 29,8 & 27,4 & 522 & 37,7 & 65,11 & 28,5 & 20 & 882 & 39,06 & 79,61 \\
\hline B & Santa Teresa & 23.392 & 21,3 & 381 & 26,39 & 26,96 & 29,3 & 604 & 39,19 & 66,3 & 30,9 & 20 & 878 & 43,91 & 83,53 \\
\hline B & $\begin{array}{l}\text { Venda Nova do } \\
\text { Imigrante }\end{array}$ & 24.800 & 17,4 & 322 & 41,36 & 38,37 & 18,1 & 559 & 103,15 & 122,99 & 23,9 & 34 & 945 & 99,01 & 146,19 \\
\hline B & Afonso Cláudio & 30.720 & 25,9 & 291 & 32,3 & 33,68 & 30,6 & 625 & 41,83 & 68,25 & 40,3 & 21 & 952 & 93,45 & 159,89 \\
\hline B & $\begin{array}{l}\text { Domingos Mar- } \\
\text { tins }\end{array}$ & 33.711 & 21,2 & 364 & 32,49 & 32,21 & 24,4 & 672 & 55,02 & 88,44 & 25,5 & 20 & 920 & 47,34 & 80,57 \\
\hline B & $\begin{array}{l}\text { Santa Maria de } \\
\text { Jetibá }\end{array}$ & 39.849 & 25 & 419 & 32,46 & 36,46 & 28 & 559 & 38,24 & 66,61 & 28,8 & 20 & 1026 & 63,92 & 109,28 \\
\hline C & Viana & 76.954 & 27,8 & 259 & 22,55 & 24,99 & 38 & 624 & 29,01 & 50,75 & 35,7 & 20 & 997 & 63,49 & 101,2 \\
\hline C & Guarapari & 122.982 & 45,7 & 349 & 39,4 & 45,49 & 38,8 & 612 & 46,13 & 72,18 & 44,3 & 38 & 1072 & 187,37 & 237,37 \\
\hline D & Cariacica & 378.603 & 29,6 & 411 & 27,2 & 28,91 & 36 & 687 & 67,04 & 84,76 & 35 & 23 & 1056 & 119,61 & 178,62 \\
\hline$D$ & Vitória & 358.267 & 42,8 & 416 & 26,21 & 31,84 & 41,3 & 655 & 44,51 & 67,54 & 40,9 & 19 & 945 & 68,1 & 126,69 \\
\hline $\mathrm{D}$ & Vila Velha & 486.208 & 40,1 & 299 & 23,82 & 25,9 & 42,2 & 665 & 33,3 & 62,59 & 43,9 & 18 & 894 & 57,91 & 122,92 \\
\hline$D$ & Serra & 507.598 & 39,9 & 335 & 19,9 & 21,16 & 42,7 & 683 & 28,09 & 59,45 & 44,7 & 14 & 877 & 53,77 & 108,15 \\
\hline \multicolumn{2}{|c|}{ RSM-ES } & 2.219 .349 & 36,9 & 419 & 25,68 & 29,34 & 38,8 & 687 & 42,72 & 70,51 & 39,8 & 20 & 1077 & 75,49 & 140,26 \\
\hline
\end{tabular}

Fonte: Elaboração própria.

A: Até 20.000 habitantes; B: 20.001 a 50.000 habitantes; C: 50.001 a 200.000 habitantes; D: Acima de 200.001 habitantes.

Para avaliar a correlação das variáveis, as análises de correlação realizadas neste estudo apontam que o tempo de espera e o porte municipal são fatores que podem influenciar o absenteísmo em consultas e exames especializados. Ao realizar o teste de correlação de Sperman, verificou-se que a taxa de absenteísmo nos três anos analisados nos 20 municípios está correlacionada significativamente com o tempo de espera máximo registrado $(\rho=0,370$; $\mathrm{p}$-valor=0,004), correlação fraca, também à média $(\rho=0,258$; $p$-valor 0,046$)$, correlação fraca, e ao porte municipal ( $\rho=0,472$; p-valor $<0,001)$ em uma correlação moderada. Essas análises são demonstradas no gráfico 1 (a, b) e no gráfico 2 . 
Gráfico 1. Diagramas de dispersão das taxas de absenteísmo em consultas e exames especializados versus maior tempo de espera (a) e tempo médio de espera (b). RSM-ES, Brasil. 2014-2016
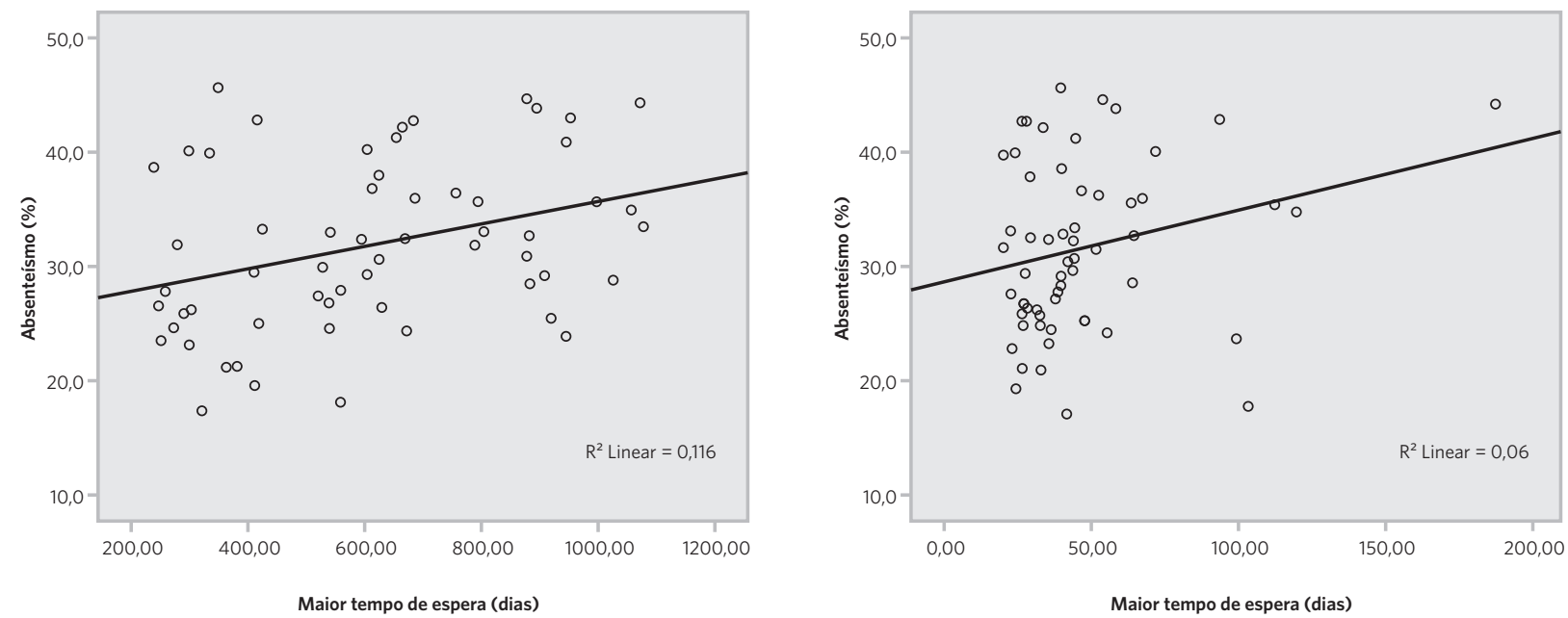

Fonte: Elaboração própria.

Gráfico 2. Box-plot entre a taxa de absenteísmo em consultas e exames especializados versus porte municipal populacional. RSM-

ES, Brasil. 2014-2016

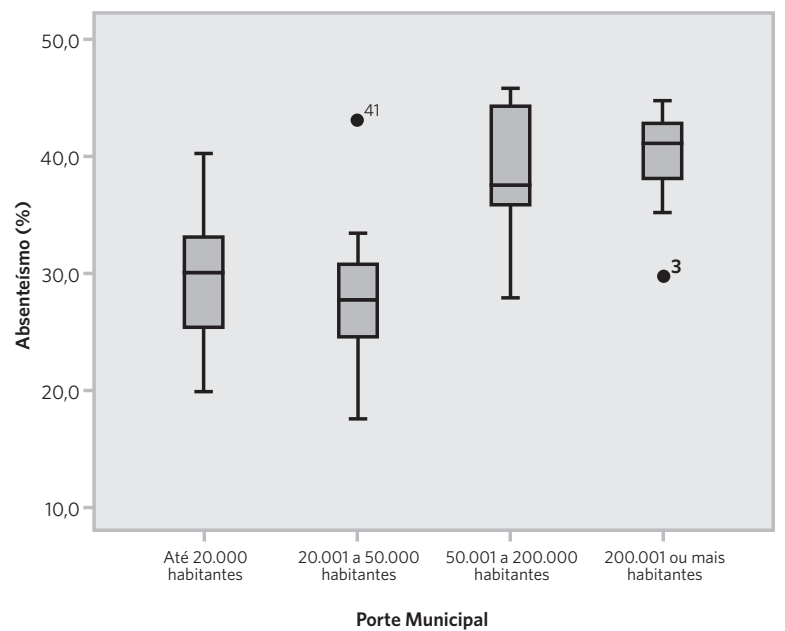

Fonte: Elaboração própria.

\section{Discussões}

O tempo de espera pelas consultas e exames especializados tem sido variável em diferentes sistemas de saúde. Na Itália, o período variou de 49 a 224 dias²1; em Ontário, Canadá, o tempo mediano foi de 41 dias ${ }^{22}$; em Teerã, Irã, o intervalo médio foi de 4 dias²3; na Alemanha, a espera média foi de 4,9 semanas, com o tempo mais longo de 5,7 semanas ${ }^{24}$, e em Ohio, EUA, houve uma espera de 4,5 semanas ${ }^{25}$.

A taxa de absenteísmo na RSM-ES nos três anos analisados foi elevada (38,6\%). Esse alto índice pode estar prolongando o tempo de 
espera pelas consultas e exames especializados, haja vista o grande número de atendimentos que deixa de ser realizado. Além disso, o tempo de espera dos usuários em consultas e exames especializados nos serviços de saúde é identificado pela Organização Mundial da Saúde (OMS) como uma das principais medidas de um sistema de saúde responsivo ${ }^{26,27}$. Um longo tempo de espera, como observado neste estudo, tem sido considerado frustrante para os usuários e parece ser uma causa potencial consistente e significativa do absenteísmo do usuário. Utentes em consultas ambulatoriais estão razoavelmente satisfeitos se não esperam muito tempo para ter acesso a essas consultas ${ }^{28}$.

Em relação ao fluxo de regulação do acesso a consultas e exames especializados, ele inicia-se nas unidades de saúde do SUS, onde o médico considera a necessidade de encaminhamento do paciente a uma avaliação especializada. A solicitação é encaminhada à Secretaria Municipal de Saúde (SMS), que cadastra o pedido no sistema informatizado, os profissionais reguladores. $\mathrm{O}$ complexo regulador é responsável pela avaliação crítica e técnica dos laudos de solicitação, pela promoção do agendamento das consultas e pelo processo de internação dos pacientes, baseado na classificação de risco, de acordo com os protocolos de regulação pactuados. Assim, os reguladores autorizam e classificam a solicitação conforme a disponibilidade de agenda. A solicitação autorizada é agendada/marcada para execução, em tese com base em algoritmo de gravidade e tempo de espera ${ }^{29}$.

Torna-se necessário destacar que, no modelo regulatório preconizado pelo modelo de atenção centrado na Atenção Primária à Saúde atuando como porta de acesso e de entrada preferencial, a unidade básica de saúde deve ser ordenadora da rede de serviços voltados à atenção e, ao mesmo tempo, ser a coordenadora do cuidado às pessoas, famílias e comunidades. Já a atenção especializada deve ser responsável por prover o cuidado complementar, no intuito garantir a integralidade, atendendo a demandas especificas e reduzindo os riscos à saúde dos usuários do sistema ${ }^{30}$. Observa-se, entretanto, que, no decorrer do processo de cuidado ao usuário, um papel frágil é conferido ao profissional da atenção básica que identificou a necessidade da consulta especializada, com elevada centralização do poder regulatório. Deve-se ressaltar também que o SUS em construção ainda convive com redes de saúde fragmentadas nas quais predomina a desarticulação entre os níveis de atenção nos seus diversos pontos, o que se transforma em uma barreira de acesso aos serviços de saúde e explica, em parte, o próprio absenteísmo ${ }^{15}$.

Quanto ao tempo de espera, ele é um indicador comumente usado pelos gestores dos serviços de saúde para avaliar o cuidado em saúde nos serviços especializados ${ }^{31}$. Estudos científicos ${ }^{32-35}$ demonstraram que o tempo de espera elevado e o histórico de absenteísmo anterior em consultas e exames previamente agendados foram determinantes do absenteísmo. Verificou-se, ainda, que o tempo mediano de espera dos usuários no presente estudo não foi muito prolongado, comparando-se com os dados de países desenvolvidos, como mediana de 17 dias no estado de New York, nos EUA, de 72 dias na Holanda e de 59 dias na Suécia ${ }^{36}$; de 5 a 6 meses na Islândia ${ }^{37}$, de 181 dias em 1993 e de 212 dias em 1995 na Nova Zelândia 38 .

As pesquisas em relação à associação entre tempo de espera e o absenteísmo do usuário na saúde ainda são escassas no Brasil. O presente estudo permitiu identificar que, no universo de dados analisados, em municípios de pequeno e grande porte, a taxa de absenteísmo aumenta ano a ano com diferença maior para as faltas em consultas em comparação aos exames, além de correlação positiva entre o tempo de espera, o absenteísmo e o porte municipal. Estudo de Simon et al. ${ }^{16}$ concorda com os resultados desta pesquisa, que indicam uma associação positiva entre o tempo de espera e o absenteísmo em consultas e exames especializados.

Além disso, estudo de Martinez et al. ${ }^{39}$ verificou uma forte correlação positiva entre o tempo de espera, fatores geográficos e a 
mortalidade em indivíduos que aguardam consultas ou exames especializados. Isso sugere que, para o objetivo de reduzir as barreiras de acesso com priorização dos casos mais graves, a variabilidade do tempo de espera em cada ponto de atenção e a regulação devem determinar o sistema de gerenciamento da lista de espera. Nesse sentido, tanto profissionais da atenção básica quanto da equipe dos Núcleos de Atenção à Saúde da Família - equipe multiprofissional - poderiam participar qualificando os referenciamentos, visto que centralizar a regulação em apenas profissionais médicos pode limitar a integralidade da atenção ao usuário. Por fim, é importante ressaltar que a redução da lista de espera deve equilibrar a necessidade de igualar a sobrevivência do usuário em curto e em longo prazo.

Verificou-se, entre as consultas e exames analisados no estudo (1.002.719), que 63.761 eram de usuários que residiam em um dos sete municípios de 20.000 habitantes, 84.167 eram de um dos sete municípios de 20.001 a 50.000 habitantes, 60.694 eram de um dos dois municípios de 50.001 a 200.000 habitantes e 795.219 eram de um dos quatro municípios acima de 200.001 habitantes. Diante desses fatos, conhecer o porte dos municípios pode auxiliar na compreensão da temática estudada. Dados do Índice de Desempenho do SUS (IDSUS), que é um indicador síntese que faz uma aferição contextualizada do desempenho do SUS quanto ao acesso (potencial ou obtido) e à efetividade do Sistema, demonstram que, nos municípios estudados, o acesso obtido na atenção ambulatorial de média complexidade não se aproxima do ideal estabelecido pelo Ministério da Saúde ${ }^{\mathbf{4 0}}$.

Aliado a esses fatos, em uma análise mais economicista, além de não garantir o acesso oportuno e prioritário para os casos com maior risco, existe ${ }^{41}$ a grande preocupação quanto à escassez de recursos financeiros na saúde para atender às necessidades em saúde que se verifica na população; o desperdício entre o número de consultas agendadas e o não comparecimento instigam ações para o enfrentamento das questões relacionadas com o crescimento da fila de espera ${ }^{\mathbf{4 2}}$. No entanto, o não comparecimento às consultas e exames é um fenômeno muito mais complexo que deve considerar como a necessidade em saúde do usuário foi transformada ao longo do tempo de espera.

É importante destacar que o absenteísmo é um fator agravante para o tempo de espera, entretanto, o agendamento de atendimento especializado pode ser afetado por vários fatores externos que não estão sob o controle do serviço de saúde ou do usuário. Estes incluem atrasos causados pela necessidade de testes diagnósticos adicionais que são realizados por terceiros ou para algumas condições; pode ser necessário ainda que vários especialistas coordenem seus cuidados para o êxito de um tratamento ${ }^{\mathbf{4 3}}$.

Assim, o tempo de espera do usuário em relação à data da solicitação até a data de execução da consulta, na grande maioria, é longo, em que muitas vezes o usuário desiste de consultar ou, por não ter alternativa, espera durante meses para a realização da consulta ${ }^{44}$. Os dados analisados permitiram observar que alguns municípios se destacaram por apresentarem tempos de espera mais longos. O tempo de espera $\mathrm{Tl}$ e a maior disponibilidade tecnológica em saúde nos municípios de grande porte parecem fazer com que a porcentagem de faltas seja maior nesses municípios ${ }^{45}$.

Entretanto, não se observou diferença significativa entre os municípios, com maior valor de T1 (409 dias) para usuários que residem em municípios de até 20.000 habitantes e 20.001 a 50.000 habitantes e 406 dias para usuários residentes em município com população acima de 200.001 habitantes no ano de 2014. A análise T1 referente ao ano de 2015 mostrou que o tempo de espera foi maior para usuários que residem em municípios com população de até 20.000 habitantes e de 20.001 a 50.000 habitantes (664 dias) e os usuários que residem em municípios com população acima de 200.001 habitantes (678 dias). No ano de 2016, os usuários que esperaram mais (1.054 dias) residiam 
em municípios com população de até 20.000 habitantes e em municípios com população de 50.001 a 200.000 habitantes (1.050 dias) respectivamente.

A variação no tempo de espera relacionada com o porte do município não fica evidente ao comparar o tempo de espera para consultas em outros estudos $\mathbf{4 4 , 4 5}^{\mathbf{4}}$, que apuraram 3,8 meses de espera para consultas especializadas nos municípios estudados, e outras médias de até 335 dias. Essa análise também não corrobora outro estudo que concluiu que usuários não residentes em municípios de maior porte apresentaram mais tempo de espera por consulta especializada ${ }^{16}$.

A reduzida governabilidade de municípios de pequeno porte sobre os serviços especializados fora de seu território pode, em parte, estar relacionada com essa diferença no acesso entre pequenos e grandes municípios, contudo, esse fato não se apresentou evidente no presente estudo ${ }^{8}$. Além disso, há o fato de não existir a garantia de que as cotas programadas pela Programação Pactuada Integrada (PPI) sejam distribuídas entre as unidades de saúde municipais tanto em pequenos como nos municípios de maior porte ${ }^{8}$.

Nesse contexto, ressalta-se que A PPI deve ser o instrumento que, em consonância com o processo de planejamento, visa definir e quantificar as ações de saúde para a população residente em cada território, além de nortear a alocação dos recursos financeiros a partir de critérios e parâmetros pactuados entre os gestores. Deve, ainda, explicitar os pactos de referência entre municípios e definir a parcela de recursos destinados à assistência da própria população e da população referenciada por outros municípios ${ }^{46}$. Isso remete à reflexão de que a descentralização e a regionalização do SUS ainda se apresentam com um desafio.

Cabe ressaltar que, sem os esforços do município para manter alguns médicos especialistas em sua rede própria de serviços de saúde, a fila de espera por consultas especializadas e, consequentemente, o tempo de espera seriam ainda maiores ${ }^{\mathbf{1 8}}$. Além disso, as maiores dificuldades referentes aos tempos de espera para a atenção especializada, ainda que o sistema funcione com territorialização, fluxos definidos e sistemas de informações compartilhados, esse nível não tem controle sobre esses processos de trabalho, prevalecendo a impressão de pouca transparência ${ }^{10}$.

Aliado a esses fatores, verificou-se no presente estudo a correlação entre o tempo de espera, o absenteísmo e o porte municipal. Nesse contexto, é importante ressaltar que o longo tempo de espera pode acarretar vários problemas para o indivíduo, para seus familiares e para a sociedade. Para o indivíduo, pode ocorrer o agravamento da doença, chegando, às vezes, ao óbito. Ademais, podem surgir problemas psicológicos e repercussões para suas famílias. Para a sociedade, quando ocorre o seu afastamento das atividades laborativas, tem como consequência a diminuição da produtividade, bem como pode acarretar custos para o pagamento de auxílio-doença; e para os que vão precocemente a óbito, há o custo social dos anos futuros perdidos de produtividade.

Para a RSM-ES, o tempo de espera gera ineficiência, além de gastos desnecessários. Apesar de iniciativas que buscam tornar mais eficiente o uso da capacidade instalada dos municípios, às vezes, esperas e atrasos relacionam-se mais aos problemas organizacionais do que à falta de recursos. Assim, torna-se de grande interesse o conhecimento do que está ocorrendo com esses usuários durante a fase de espera.

\section{Conclusões}

Absenteísmo em consultas e exames especializados é um tema atual e relevante. Avaliar e monitorar o tempo de espera e conhecer como certos fatores impactam o absenteísmo por consultas/exames especializados, em sua maioria de usuários que residem distante de grandes centros urbanos, podem substanciar mudanças nas políticas de agendamento de consultas/exames especializados. 
O estudo demonstrou que existe uma correlação positiva entre o tempo de espera e o absenteísmo e o porte municipal. De modo geral, o tempo de espera, que envolve o processo regulatório de agendamento das consultas e exames especializados, é fator importante que demonstrou influenciar o absenteísmo. Além disso, o tempo de espera entre a solicitação e o agendamento e a maior disponibilidade tecnológica em saúde nos municípios de grande porte parecem fazer com que o absenteísmo seja maior nesses municípios.

Os resultados deste estudo são relevantes por ser um estudo primário abrangente. Embora seja difícil projetar o cálculo do tempo médio de espera ideal por atendimento especializados, a partir das análises, existe a possibilidade de desenvolver estratégias com base nas características da população das áreas envolvidas e da referência histórica da intensidade de utilização (determinado pelo número de consultas por habitante). Isso, além de interesse para os gestores, é importante para a sociedade, já que o tempo de espera é uma das variáveis associadas à compra de serviços privados de saúde, o que supõe um custo econômico adicional à gestão da saúde.

O presente estudo tem as suas limitações, entretanto, cremos não ter havido vieses evidentes em relação a esta investigação, que é a primeira a apresentar dados sobre tempos de espera nos ${ }^{\mathbf{2 0}}$ municípios da RSM-ES, com dados analisados ao longo de um período de três anos. $\mathrm{O}$ estudo analisou o tempo de espera por consulta e exames especializados em pequenos municípios e grandes municípios relativos a usuários que faltaram aos procedimentos agendados.

\section{Colaboradores}

Farias CML (0000-0001-5826-5261)* participou do planejamento, concepção, metodologia e redação final da metassíntese. Giovanella $\mathrm{L}$ (0000-0002-6522-545X)* teve contribuição substancial na revisão crítica do artigo. Oliveira AE (0000-0002-9679-8592)* contribuiu significativamente no planejamento e redação final do artigo. Santos Neto ET (0000-0002-73517719)* participou na concepção, metodologia, redação final do artigo. 


\section{Referências}

1. Olayiwola JN, Anderson D, Jepeal N, et al. Electronic consultations to improve the primary care- Specialty care interface for cardiology in the medically underserved: A cluster-randomized controlled trial. Ann fam med. 2016; 14(2):133-140.

2. Carrière G, Sanmartin C. Waiting time for medical specialist consultations in Canada, 2007. Health rep. 2010; 21(2):7-14.

3. Dall TM, Gallo PD, Chakrabarti R, et al. An Aging population and growing disease burden will require a large and specialized health care workforce by 2025 . Health aff. 2013; 32(11):2013-2020.

4. Keely E, Liddy C, Afkham A. Utilization, benefits, and impact of an e-Consultation service across diverse specialties and primary care providers. Telemed j e health. 2013; 19(10):733-738.

5. Hurst J, Siciliani L. Tackling excessive waiting times for elective surgery: a comparison of policies in twelve OECD countries. Paris: OECD; 2003.

6. Schoen C, Osborn R, Huynh PT, et al. Primary care and health system performance: adults' experiences in five countries. Health aff. 2004; Suppl. Web Exclusives (W4): 487-503.

7. Conselho Nacional de Secretários de Saúde. A saúde na opinião dos brasileiros. Brasília, DF: Conass; 2003.

8. Giovanella L, Mendonça MHM, Almeida PF, et al. Saúde da Família: Limites e possibilidades para uma abordagem integral de atenção primária à saúde no Brasil. Ciênc. Saúde Colet. 2009; 14(3):783-794.

9. LaGanga LR, Lawrence S. Clinic Overbooking to Improve Patient Access and Increase Provider Productivity. Decision Sciences. 2007; 38(2):251-276.

10. Conill EM, Giovanella L, Almeida PF. Listas de espera em sistemas públicos: da expansão da oferta para um acesso oportuno? Considerações a partir do Sis- tema Nacional de Saúde espanhol. Ciênc. Saúde Colet. 2011; 16(6):2783-2794.

11. Aeenparast A, Farzadi F, Maftoon F. Waiting time for specialist consultation in tehran. Arch Iran med. 2012; 15(12):756-758.

12. Peng Y, Erdem E, Shi J, et al. Large-scale assessment of missed opportunity risks in a complex hospital setting. Inform Health Soc Care. 2016; 41:112-127.

13. Barbosa DVS, Barbosa NB, Najberg E. Regulação em Saúde: desafios à governança do SUS. Cad. Saúde Coletiva. 2016; 1(24):49-54.

14. Vieira J. Listas de espera para cuidados de saúde: onde fica a ética? Nursing. 2007; 17(217):30-35.

15. Spedo SM, Pinto NRS, Tanaka OY. O difícil acesso a serviços de média complexidade no SUS: o caso da cidade de São Paulo, Brasil. Physis. 2010; 20(3):953972

16. Simon JC, Maltchik M, Silva EE, et al. Avaliação do tempo de espera para consultas de reumatologia em um centro de atendimento terciário de Porto Alegre - RS. Rev AMRIGS. 2008; 52(4):303-308.

17. Regidor E, Martínez D, Astasio P, et al. Asociación de los ingresos económicos con la utilización y la accesibilidad de los servicios sanitarios en España. Gac. sanit. (Barc., Ed. impr.). 2006; 20(5):352-359.

18. Vieira EWR, Lima TMN, Gazzinelli A. Tempo de espera por consulta médica especializada em um município de pequeno porte de Minas Gerais, Brasil. REME rev. min. enferm. 2015; 19(1):65-71

19. Brasil. Instituto Brasileiro de Geografia e Estatística - IBGE. Síntese de indicadores sociais: uma análise das condições de vida da população brasileira: 2017, Coordenação de População e Indicadores Sociais. Rio de Janeiro: IBGE; 2017. 
20. Brasil. Instituto Brasileiro de Geografia e Estatística - IBGE. Cidades e Estados. Brasil em Síntese. Rio de Janeiro: IBGE; 2018.

21. Deluca J, Goldschmidt A, Eisendle K. Requests for dermatology specialist consultations show an inverse correlation with waiting time: an analysis of waiting time to access dermatology specialist health care in Bolzano, South Tyrol, Italy. Br j dermatol. 2015; 172(4):1133-1135.

22. Jaakkimainen L, Glazier R, Barnsley J, et al. Waiting to see the specialist: patient and provider characteristics of wait times from primary to specialty care. BMC Fam Pract. 2014; 15(1):15-16.

23. Aeenparast A, Maftoon F, Farzadi F, et al. Waiting Time for First Outpatient Visit in Specialty Level: Assessing the Provider Related Factors. Arch Iran med. 2015; 18(3):185-188.

24. Krensel M, Augustin M, Rosenbach T, et al. Waiting time and practice organization in dermatology. J. Dtsch. Dermatol. Ges. 2015; 13(80):812-814.

25. Uhlenhake E, Brodell R, Mostow E. The dermatology work force: A focus on urban versus rural wait times. J. Am. Acad. Dermatol. 2009; 61(1):17-22.

26. Valentine NB, Silva A, Kawabata K, et al. Responsiveness of the health system: concepts, domains and operationalization. In: Murray CJL, Evans DB, et al., editores. Evaluation of health systems performance: debates, methods and empiricism. Geneva: World Health Organization; 2003. p. 96.

27. Silva A. A framework for measuring responsiveness. Series of GPE Discussion Papers: n. 32. EIP / GPE / EBD. Geneva: World Health Organization; 2010

28. Huang YL, Hanauer DA. Patient no-show predictive model development using multiple data sources for an effective overbooking approach. Appl Clin Inform. 2014; 5(3):836-860.

29. Santos FP, Merhy EE. Public regulation of the heal- th care system in Brazil - a review. Interface (Botucatu). 2006; (10)19:25-41.

30. Brasil. Ministério da Saúde, Secretaria de Atenção à Saúde, Departamento de Atenção Básica. Política Nacional de Atenção Básica. Brasília, DF: Ministério da Saúde; 2006.

31. Bittar OJN, Magalhães A, Martines CM, et al. Absenteísmo em atendimento ambulatorial de especialidades no estado de São Paulo. BEPA, Bol. epidemiol. paul. (Impr.). 2016; 13(152):19-32.

32. Parikh A, Gupta K, Wilson AC, et al. The effectiveness of outpatient appointment reminder systems in reducing no-show rates. Am j med. 2010; 123(6):542548.

33. Gupta D, Wang WY. Patient appointments in ambulatory care. In: Hall R, editor. Handbook of healthcare system scheduling. New York: Springer; 2012.

34. Cavalcante RP, Cavalcanti JCM, Serrano RMSM, et al. Absenteísmo de consultas especializadas no sistema de saúde público: relação entre causas e o processo de trabalho de equipe de saúde da família, João Pessoa - PB, Brasil. Tempus. 2013; 7(2):63-84.

35. Dantas RAN, Torres GV, Salvetti MG, et al. Avaliação da satisfação dos usuários atendidos por serviço pré-hospitalar móvel de urgência. Saúde debate. 2017; 20(230):1796-1800.

36. Berstein SJ, Rigter H, Brorsson B, et al. Waiting for coronary revascularization: a comparison between New York State, The Netherlands and Sweden. Health Policy. 1997; 42(1):15-27.

37. Jonsdottir H, Baldursdottir L. The experience of people awaiting coronary artery bypass graft surgery: the Icelandic experience. J adv. nurs 1998; 27(1):68-74.

38. Martinez DA, Zhang H, Bastias M, et al. Prolonged wait time is associated with increased mortality for Chilean waiting list patients with non-prioritized conditions. BMC public health. 2019; 19(1):233. 
39. Doogue M, Brett C, Elliot JM. Life and death on the waiting list for coronary bypass surgery. $\mathrm{N} \mathrm{Z}$ med j. 1997; 110(1037): 26-30.

40. Brasil. Ministério da Saúde, Secretaria Executiva, Departamento de Monitoramento e Avaliação do SUS (IDSUS). Brasília, DF: Ministério da Saúde; 2015.

41. Barbosa DVS, Barbosa NB, Najberg E. Regulação em Saúde: desafios à governança do SUS. Cad. Saúde Coletiva. 2016; 24(1):49-54.

42. Albieri FAO, André LDSM, Malaquias AK, et al. Gestão de fila de espera da atenção especializada ambulatorial: resgatando o passado e buscando um novo olhar para o futuro. São Paulo: Secretaria Municipal de Saúde; 2014.

43. Murray M. Reduzindo esperas e atrasos no processo de encaminhamento. Gestão de Práticas Familiares. 2002; 9(3):39-42.
44. Bender AS, Molina LR, Mello ALSF. Absenteísmo na atenção secundária e suas implicações na atenção básica. Espaç. saúde. 2010; 11(2):56-65.

45. Guerra Jr AA, Acúrcio FA, Gomes CAP, et al. Disponibilidade de medicamentos essenciais em duas regiões de Minas Gerais, Brasil. Rev. panam. salud pública. 2004; 15(3):168-175.

46. Brasil. Ministério da Saúde, Secretaria de Atenção à Saúde, Departamento de Regulação. Avaliação e Controle de Sistemas. Diretrizes para a programação pactuada e integrada da assistência à saúde. Brasília, DF: Ministério da Saúde; 2006.

Recebido em 26/04/2019

Aprovado em 05/11/2019

Conflito de interesses: inexistente

Suporte financeiro: Fundação Amparo à Pesquisa do Estado do

Espírito Santo 